\title{
Knockdown of interferon-stimulated gene 15 affects the sensitivity of hepatocellular carcinoma cells to norcantharidin
}

\author{
BAOXIANG CHEN ${ }^{1,2}$, SHUQIANG JIN ${ }^{3}$, BIN BAI $^{3}$, ZHI LI $^{1}$, CAIFANG NI $^{1}$ and YANSEN LIU ${ }^{2}$ \\ ${ }^{1}$ Department of Interventional Radiology, The First Affiliated Hospital of Soochow University, Suzhou, Jiangsu 215006; \\ ${ }^{2}$ Department of Interventional Radiology, Inner Mongolia Forestry General Hospital, The Second Clinical Medical School of \\ Inner Mongolia University for The Nationalities, Yakeshi, Inner Mongolia 022150; ${ }^{3}$ Department of Interventional Radiology, \\ The Second Affiliated Hospital of Harbin Medical University, Harbin, Heilongjiang 150086, P.R. China
}

Received September 10, 2018; Accepted June 20, 2019

DOI: $10.3892 /$ etm.2019.8028

\begin{abstract}
Interferon-stimulated gene 15 (ISG15) serves a crucial role in hepatocellular carcinoma (HCC) progression. The present study explored the effect of ISG15 knockdown on the sensitivity of HCC cells to norcantharidin. The expression of ISG15 in HCC tissues and cell lines was assessed by reverse transcription-quantitative polymerase chain reaction and immunohistochemistry. Pearson's $\chi^{2}$ test was conducted to analyze the correlation between the clinicopathological features and ISG15 expression of patients with HCC. In addition, HCC cells were transfected with small interfering RNA against ISG15, ISG15 overexpression plasmid or respective negative controls. Cell proliferation, clonogenic ability and apoptosis were examined by Cell Counting Kit-8, colony formation and Annexin V/propidium iodide staining assays, respectively. Protein expression was assessed by western blot analysis. The results revealed that ISG15 was overexpressed in HCC tissues, and that ISG15 expression was positively correlated with HCC differentiation and metastasis. Downregulation of ISG15 increased the sensitivity of HCC cells to norcantharidin, and norcantharidin treatment reversed the tumor-promoting effects of ISG15 overexpression exerted in HCC cells. Furthermore, the expression levels of apoptosis-associated proteins were regulated by ISG15 and norcantharidin. Taken together, the observed increase in the sensitivity of HCC cells to norcantharidin was facilitated by ISG15 knockdown and may provide novel insights for HCC therapy.
\end{abstract}

Correspondence to: Dr Caifang Ni, Department of Interventional Radiology, The First Affiliated Hospital of Soochow University, 889 Pinghai Road, Suzhou, Jiangsu 215006, P.R. China

E-mail: caifeng_nizhsohu@sohu.com

Key words: interferon-stimulated gene 15, liver cancer, hepatocellular carcinoma, apoptosis, norcantharidin

\section{Introduction}

Hepatocellular carcinoma (HCC) is one of the most common and malignant tumors worldwide, and mortality associated with $\mathrm{HCC}$ is the fourth highest in the world (1). The pathogenesis of HCC is unclear, early diagnosis is difficult and the tumor develops rapidly $(2,3)$. The majority of patients are at later or advanced stages of the disease at the time of diagnosis, and thus few patients are eligible to undergo radical surgical resection (4,5). Resection rate is low and the degree of recurrence is high for HCC patients. Other treatments, including radiotherapy, chemotherapy and interventional therapy, have side effects and a limited influence on inhibiting the recurrence and metastasis of $\mathrm{HCC}(6,7)$. Therefore, it is urgent to seek novel and effective therapeutic drugs.

Norcantharidin increases the number of white blood cells, as well as inhibits the proliferation, invasion and metastasis, and induces apoptosis in tumor cells. This agent is often used in the treatment of various tumor types, including $\operatorname{HCC}(8,9)$. Yeh et al (10) demonstrated that norcantharidin exhibits antimetastatic effects on HCC by transcriptional inhibition of matrix metalloproteinase- 9 through the modulation of nuclear factor- $\kappa \mathrm{B}$ activity. In addition, Sun et al (11) reported that norcantharidin treatment induces autophagic cell death in HCC cells. A study by Gao et al (12) also indicated that norcantharidin inhibits interleukin-6-induced epithelial-mesenchymal transition via the JAK2/STAT3/TWIST signaling pathway in HCC cells.

Interferon-stimulated gene 15 (ISG15), a ubiquitin-type molecule, is strongly upregulated by type I interferons as a primary response to diverse microbial and cellular stress stimuli (13). ISG15 expression is increased in various cancer types and is considered as a potential prognostic marker (14-16). Additionally, ISG15 is overexpressed in $\mathrm{HCC}$ and acts as a trigger for tumorigenesis and metastasis $(17,18)$. Desai et al (19) demonstrated that ISG15 is a potential tumor biomarker for camptothecin (CPT) sensitivity. Tessema et al (15) reported that ISG15 sensitizes non-small cell lung cancer cells to topoisomerase-1 inhibitor and CPT treatment. However, the effect of ISG15 on the sensitivity of HCC cells to norcantharidin remains to be established.

In the present study, the objective was to examine the association between norcantharidin and ISG15 expression 
on the viability of HCC. ISG5 expression was examined in clinical HCC samples, and it was demonstrated that aberrant ISG15 expression in HCC tissues was correlated with multiple malignant clinicopathological characteristics. Furthermore, ISG15 knockdown sensitized HCC cells to apoptosis induced by norcantharidin.

\section{Materials and methods}

Patients and tissue samples. Paired HCC tissues $(\mathrm{n}=37)$ and matched non-tumor samples were obtained from a tissue bank of samples collected from patients that underwent surgical treatment between May 2010 and March 2016 at The First Affiliated Hospital of Soochow University (Suzhou, China). Patient characteristics are listed in Table I. The clinicopathological significance of ISG15 levels in patients with HCC was studied according to methods described in the previous studies (20-22). Following surgical resection, the tissue samples were immediately snap frozen in liquid nitrogen and stored at $-80^{\circ} \mathrm{C}$. Patients that had received radiotherapy and/or immunotherapy prior to surgical treatment were excluded from the current study. Written informed consent was obtained from all patients. The study protocol conformed to the ethical guidelines of the 1975 Declaration of Helsinki and was approved by The First Affiliated Hospital of Soochow University.

Immunohistochemical (IHC) analysis. ISG15 protein expression in 37 paired paraffin-embedded tumor and adjacent normal tissues was detected using an immunoperoxidase method. In brief, following deparaffinization of samples, antigen retrieval was performed in a sodium citrate solution ( $\mathrm{pH}$ 6.0). Tumor and adjacent normal tissues were cut into $3-\mu \mathrm{m}$-thick sections, and the sections were blocked with normal goat serum at $37^{\circ} \mathrm{C}$ for $60 \mathrm{~min}$ and incubated with rabbit anti-ISG15 antibody (1:100; cat. no. ab131119; Abcam) overnight at $4^{\circ} \mathrm{C}$. Subsequently, sections were washed and incubated with horseradish peroxidase (HRP)-conjugated anti-rabbit IgG antibody $(1: 1,000$; cat. no. ab6721; Abcam) at room temperature for $1 \mathrm{~h}$. Finally, 3,3'-diaminobenzidine tetrahydrochloride (DAB; ZSGB-BIO; OriGene Technologies, Inc.) was used as a substrate to visualize the bound antibody for $30 \mathrm{~min}$ at $20^{\circ} \mathrm{C}$, following which the sections were counterstained with hematoxylin for $10 \mathrm{~min}$ at $20^{\circ} \mathrm{C}$, dehydrated with an ascending series of ethanol concentrations and mounted with neutral resin. The samples observed with a light microscope (magnification, x400).

Cell lines. Four HCC cell lines Hep3B, Huh7, MHCC97-H and MHCC97-L $(23,24)$ were purchased from the Type Culture Collection of the Shanghai Institute of Biochemistry and Cell Biology, Chinese Academy of Sciences (Shanghai, China). Cells were maintained in Dulbecco's modified Eagle's medium (DMEM; Gibco; Thermo Fisher Scientific, Inc.) supplemented with $10 \%$ fetal bovine serum (FBS; Gibco; Thermo Fisher Scientific, Inc.), $100 \mathrm{U} / \mathrm{ml}$ penicillin and $100 \mu \mathrm{g} / \mathrm{ml}$ streptomycin (Sigma-Aldrich; Merck KGaA) at $37^{\circ} \mathrm{C}$ with $5 \% \mathrm{CO}_{2}$.

Cell transfection. For ISG15 knockdown, small interfering RNA (siRNA) targeting ISG15 (referred to as siISG15) was purchased from Shanghai GenePharma Co., Ltd., and its sequence was as follows: 5'-TGAGCACCGTGTTCATGAATC
TGCG-3'. A negative control siRNA (siNC) was also obtained, as follows: 5'-UCUCCGAACGUGUCACGUTT-3'. HCC cells were transfected with siISG15 or siNC using Lipofectamine 2000 (Invitrogen; Thermo Fisher Scientific, Inc.) following the manufacturer's protocols. For overexpression of ISG15, human ISG15 was cloned into a pcDNA3.1 expression vector. The ISG15 expression vector or control vector (NC) was then transfected into the cells using Lipofectamine 2000, according to the manufacturer's protocol. In order to confirm the knockdown and overexpression, ISG15 mRNA and protein expression levels were detected by reverse transcription-quantitative polymerase chain reaction (RT-qPCR) and western blot analysis, respectively.

$R T-q P C R$. The expression of ISG15 was evaluated by RT-qPCR. Briefly, cells were seeded in 6-well plates at a density of $5 \times 10^{5}$ cells/well, cultured overnight and then subjected to transfection for $48 \mathrm{~h}$. Total RNA was extracted from the clinical specimens and cells using TRIzol ${ }^{\circledR}$ reagent (Invitrogen; Thermo Fisher Scientific, Inc.) according to the manufacturer's protocol. The total RNA was then reverse transcribed into cDNA using the TransCript One-step gDNA Removal and cDNA Synthesis SuperMix (TransGen Biotech Co., Ltd.). ISG15 expression in cells was detected by qPCR using SYBR Green I chemistry (TransStart Top Green qPCR SuperMix; TransGen Biotech Co., Ltd.). The cycling parameters defined as follows: Initial cycling for $5 \mathrm{~min}$ at $95^{\circ} \mathrm{C}$, followed by 40 cycles at $95^{\circ} \mathrm{C}$ for $15 \mathrm{sec}, 60^{\circ} \mathrm{C}$ for $30 \mathrm{sec}$ and $72^{\circ} \mathrm{C}$ for $30 \mathrm{sec}$. The primers used for the amplification of the indicated genes were designed using the Primer Express Software (Applied Biosystems; Thermo Fisher Scientific, Inc.), and were as follows: RPN2 forward, 5'-GGACCTGACGGT GAAGATG-3', and reverse, 5'-TGGTGTTCCGAAGTTGGT CA-3' (product, 142 bp); GAPDH forward, 5'-ACCCAGAAG ACTGTGGATGG-3', and reverse, 5'-TCAGCTCAGGGATG ACCTTG-3' (product, 124 bp). The relative expression level was calculated according to the $2^{-\Delta \Delta \mathrm{Cq}}$ method, where $\Delta \mathrm{Cq}=\mathrm{Cq}$ (gene of interest)-Cq (housekeeping gene) (25). GAPDH served as an internal control. All RT-qPCR reactions were performed in triplicate.

Cell proliferation assay. Cells were seeded at a density of 5,000 cells/well in 96-well plates at $24 \mathrm{~h}$ after transfection and then treated with norcantharidin (CAS: 5442-12-6; $40 \mu \mathrm{g} / \mathrm{ml}$; Shanghai Yuanye Biotechnology Co., Ltd.) or DMSO. Cell proliferation was assessed using a Cell Counting Kit-8 (CCK-8; Dojindo Molecular Technologies, Inc.) assay at 24, 48 and $72 \mathrm{~h}$ following seeding. Absorbance was determined at $450 \mathrm{~nm}$ using a microplate spectrophotometer.

Colony formation assay. Colony formation assays were used to assess the clonogenic ability of HCC cells. After $24 \mathrm{~h}$ transfection and then $48 \mathrm{~h}$ treatment with norcantharidin, cells ( $2 \times 10^{3} /$ well) were trypsinized into single-cell suspensions and seeded in 6-well dishes. Subsequently, cells were maintained in DMEM supplemented with $10 \%$ FBS for 2 weeks. Subsequently, visible colonies were fixed in $4 \%$ paraformaldehyde for $4 \mathrm{~h}$ at $37^{\circ} \mathrm{C}$ and stained with $0.5 \%$ crystal violet for $2 \mathrm{~h}$ at $37^{\circ} \mathrm{C}$ (Beyotime Institute of Biotechnology). Colony numbers were counted under a light microscope. 
Table I. Association between ISG15 expression and the clinicopathological features of hepatocellular carcinoma patients.

\begin{tabular}{|c|c|c|c|}
\hline \multirow[b]{2}{*}{ Features } & \multicolumn{2}{|c|}{$\begin{array}{c}\text { ISG15 } \\
\text { expression (n) }\end{array}$} & \multirow[b]{2}{*}{$\mathrm{P}$-value } \\
\hline & Low & High & \\
\hline Cases (n) & 15 & 22 & \\
\hline Age (years) & & & 0.516 \\
\hline$<60$ & 12 & 7 & \\
\hline$\geq 60$ & 9 & 9 & \\
\hline Gender & & & 0.674 \\
\hline Male & 11 & 9 & \\
\hline Female & 10 & 7 & \\
\hline$\alpha$-fetoprotein $(\mu \mathrm{g} / \mathrm{l})$ & & & 0.33 \\
\hline$<400$ & 9 & 9 & \\
\hline$\geq 400$ & 6 & 13 & \\
\hline Size (cm) & & & 0.026 \\
\hline$<3$ & 11 & 8 & \\
\hline$\geq 3$ & 4 & 14 & \\
\hline TNM stage & & & 0.014 \\
\hline $\mathrm{I} / \mathrm{II}$ & 12 & 8 & \\
\hline III/IV & 3 & 14 & \\
\hline Differentiation degree & & & 0.033 \\
\hline Moderate to well & 10 & 17 & \\
\hline Poor & 5 & 5 & \\
\hline
\end{tabular}

Mean expression was used as the cutoff value. For analysis of the association between ISG15 levels and clinical features, Pearson's $\chi^{2}$ test was conducted. TNM, tumor node metastasis.

Cell apoptosis assay. Cells were harvested, washed with ice-cold PBS and stained with Annexin V-fluorescein isothiocyanate (FITC)/propidium iodide (PI) apoptosis detection kits (Nanjing KeyGen Biotech Co., Ltd.) according to the manufacturer's protocol. Cell apoptosis was measured using a flow cytometer, and the results were analyzed with FlowJo software, version 7.6.1 (FlowJo LLC).

Western blot analysis. HCC cells were dissociated using the Total Protein Extraction kit (Wuhan Goodbio Technology Co., Ltd.) according to the manufacturer's instructions, and the extracted protein was examined by western blotting. Protein concentrations were assessed using a bicinchoninic acid assay prior to loading. Total protein lysates $(40 \mu \mathrm{g}$ protein/lane) were resolved by SDS-PAGE on $10 \%$ gels and then transferred to polyvinyl difluoride membranes (EMD Millipore). Following blocking in TBS containing $0.1 \%$ Tween-20 (TBS-T) with 5\% nonfat dry milk for $30 \mathrm{~min}$ at $37^{\circ} \mathrm{C}$, the membranes were washed with TBS-T (4X) and incubated overnight at $4^{\circ} \mathrm{C}$ with primary antibodies. All the primary antibodies used in this experiment were obtained from Abcam and were as follows: Anti-caspase-9 (1:800; cat. no. ab202068), anti-caspase-3 (1:500; cat. no. ab13847), anti-GAPDH (1:1,000; cat. no. ab5174), anti-B-cell lymphoma 2 (Bcl-2; 1:1,000; cat. no. 196495), anti-Bcl-2-like protein 4 (Bax; 1:1,000; cat. no. ab32503) and anti-ISG15 (1:2,000; cat. no. ab131119). Following extensive washing, the membranes were incubated with polyclonal HRP-conjugated goat anti-rabbit IgG secondary antibody (cat. no. 7074; CST Biological Reagents Co., Ltd.) at a dilution of 1:2,000 for $1 \mathrm{~h}$ at $25^{\circ} \mathrm{C}$. Immunoreactivity was detected with an enhanced chemiluminescence reagent (Pierce; Thermo Fisher Scientific, Inc.), and subsequently visualized using a ChemiDoc XRS imaging system and analysis software (Bio-Rad Laboratories, Inc.). GAPDH served as the internal loading control.

Statistical analysis. Data from three independent experiments are expressed as the mean \pm standard deviation. Statistical analysis was performed using SPSS software, version 19.0 (IBM Corp.). Student's t-test was used to compare the mean values between two groups, while one-way analysis of ANOVA with Dunnett's multiple comparisons test was used to compare the mean values among multiple groups. Pearson's $\chi^{2}$ test was conducted for correlation analysis between the clinicopathological features and ISG15 expression of patients with HCC. $\mathrm{P}<0.05$ was considered to indicate a statistically significant difference.

\section{Results}

ISG15 is overexpressed in HCC tissues and cell lines. To identify the biological role of ISG15 in HCC, the expression of ISG15 was detected in 37 paired HCC and normal tissues. As presented in Fig. 1A, the expression of ISG5 was significantly increased in HCC tissues, as compared with that in normal tissues $(\mathrm{P}<0.001)$. The protein expression of ISG15 was examined by IHC analysis, and representative images are presented in Fig. 1B. It was observed that ISG15 was evidently overexpressed in HCC tissues as compared with the adjacent non-tumor tissues (Fig. 1B). Additionally, the mRNA levels of ISG15 in a number of HCC cell lines, including Hep3B, Huh7, MHCC97-H and MHCC97-L were evaluated by RT-qPCR. Among the HCC cell lines assessed, Huh7 cells exhibited the highest expression of ISG15, while Hep3B cells exhibited the lowest expression of ISG15. For this reason, downregulation of ISG15 was performed in Huh7 cells, whereas overexpression of ISG15 was performed in Hep3B cells.

As described in previous studies (21-23), the clinicopathological significance of ISG15 levels in patients with HCC was further investigated. The 37 patients were divided into two subgroups based on the mean expression value, which included the low $(n=15)$ and high $(n=22)$ ISG15 expression groups. As presented in Table I, ISG15 levels in HCC tissues were positively correlated with the tumor size, TNM stage and tumor differentiation $(\mathrm{P}<0.05)$. Taken together, these results indicated that ISG15 may function as an oncogene in HCC.

Downregulation of ISG15 increases the sensitivity of HCC cells to norcantharidin. To identify the effect of ISG15 on the sensitivity of HCC to norcantharidin, Huh7 cells were transfected with siISG15 and then treated with norcantharidin. The expression of ISG15 in Huh7 cells was assessed by RT-qPCR and western blot analysis, confirming that downregulation was successfully established in the siISG15 group (Fig. 2A and B). 
A

B
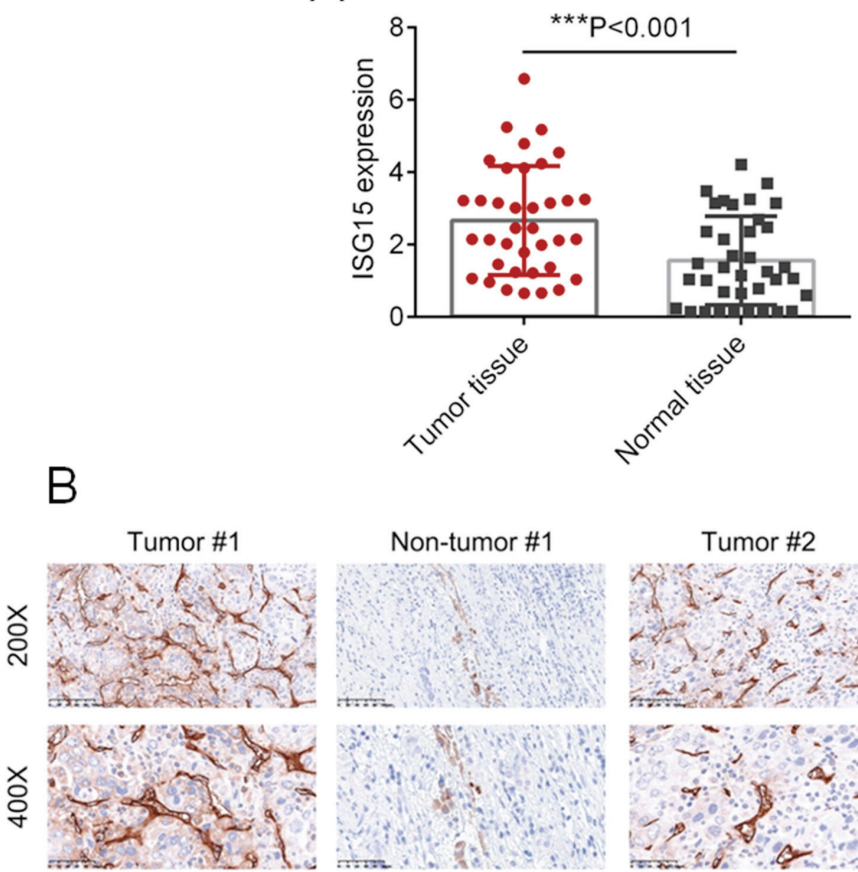

Tumor \#2

Non-tumor \#2

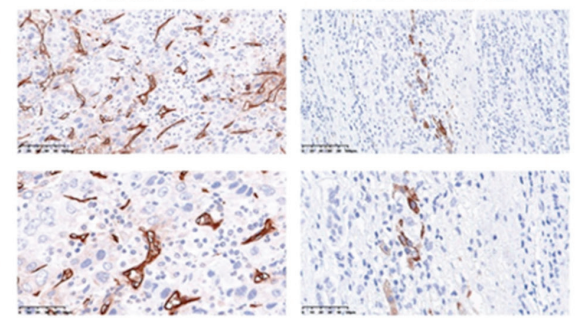

C

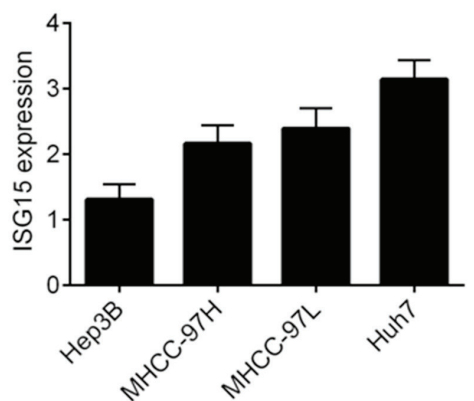

Figure 1. Expression of ISG15 is increased in HCC tissues. (A) mRNA and (B) protein expression of ISG15 in tumor and adjacent normal tissues from HCC patients was assessed by RT-qPCR and immunohistochemical analysis, respectively. (C) mRNA expression of ISG15 in Hep3B, Huh7, MHCC97-H, and MHCC97-L was determined by RT-qPCR. Data are presented as the mean \pm standard deviation. ISG15, interferon-stimulated gene 15; HCC, hepatocellular carcinoma; RT-qPCR, reverse transcription-quantitative polymerase chain reaction.

Next, cell proliferation and apoptosis were measured by CCK-8, colony formation and Annexin V/PI staining assays. The results revealed that cell proliferation and colony formation were significantly decreased in cells treated with siISG15 and/or norcantharidin, with the combination of siISG15 and norcantharidin leading to the lowest cell viability and number of colonies ( $\mathrm{P}<0.01$; Fig. $3 \mathrm{C}$ and $\mathrm{D})$. Annexin V/PI staining demonstrated that treatment with siISG15 and/or norcantharidin significantly induced apoptosis in Huh7 cells $(\mathrm{P}<0.01$; Fig. 3E). Combined treatment with siISG15 and norcantharidin significantly promoted apoptosis when compared with the siISG15 or norcantharidin single treatments $(\mathrm{P}<0.01$; Fig. 3E). Taken together, these results suggested that downregulation of ISG15 may promote the antitumor effect of norcantharidin on Huh7 cells.

Norcantharidin reverses the tumor-promoting effects of ISG15 in HCC cells. To investigated whether norcantharidin reverses the tumor-promoting effects of ISG15 in HCC cells, Hep3B cells were transfected with an ISG15 overexpression plasmid and then treated with norcantharidin. The transfection efficiency was measured using RT-qPCR and western blot analysis. It was revealed that ISG15 was significantly overexpressed in Hep3B cells transfected with the plasmid, as compared with the control group ( $\mathrm{P}<0.001 ;$ Fig. $3 \mathrm{~A}$ and $\mathrm{B})$. Cell proliferation and apoptosis were then analyzed. As presented in Fig. 3C and D, ISG15 overexpression alone significantly increased the cell proliferation and colony formation ability compared with the control group $(\mathrm{P}<0.001)$. By contrast, treatment with norcantharidin resulted in marked reduction in cell proliferation and colony formation compared with the control $(\mathrm{P}<0.001)$. However, the combination treatment significantly reduced the norcantharidin-induced inhibition of cell proliferation and colony formation, suggesting that ISG15 overexpression may reverse the antiproliferative effect of norcantharidin $(\mathrm{P}<0.001$; Fig. $3 \mathrm{C}$ and $\mathrm{D})$. Furthermore, the results of Annexin V/PI staining analysis revealed that norcantharidin induced marked cell apoptosis $(\mathrm{P}<0.001$; Fig. $3 \mathrm{E})$, while ISG15 overexpression reversed the effect of norcantharidin on the apoptosis of Hep3B cells $(\mathrm{P}<0.001$; Fig. 3E). 

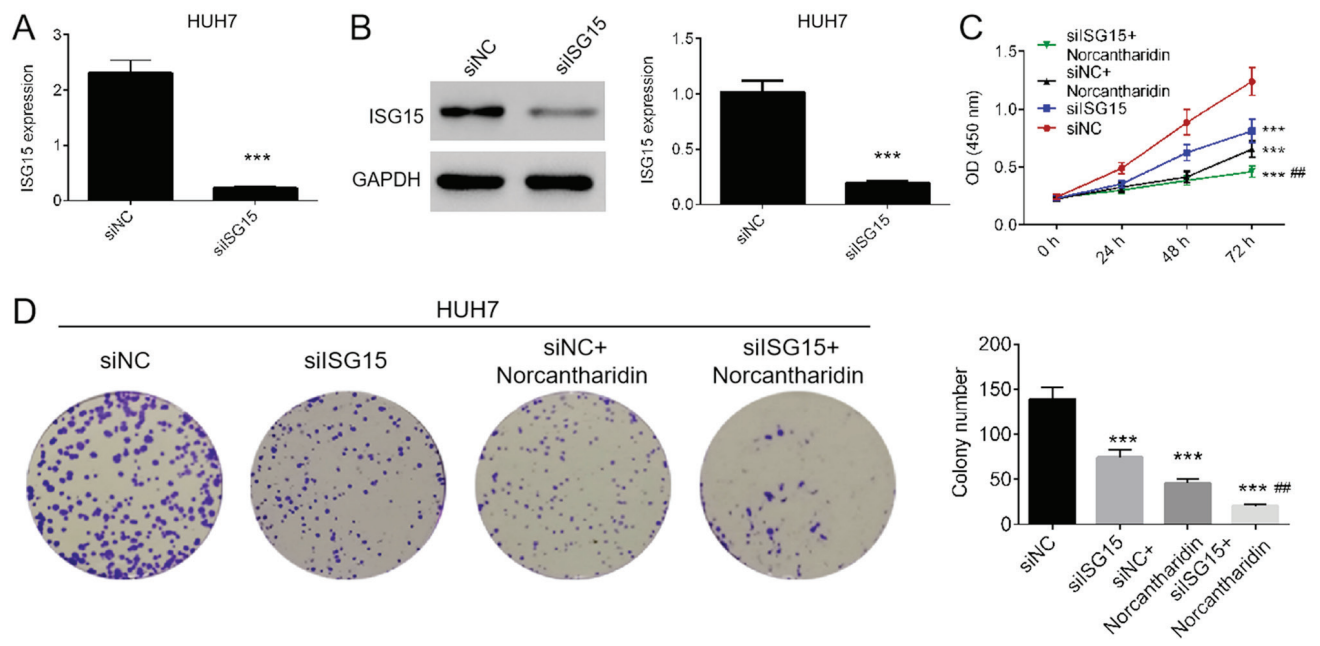

$\mathrm{E}$
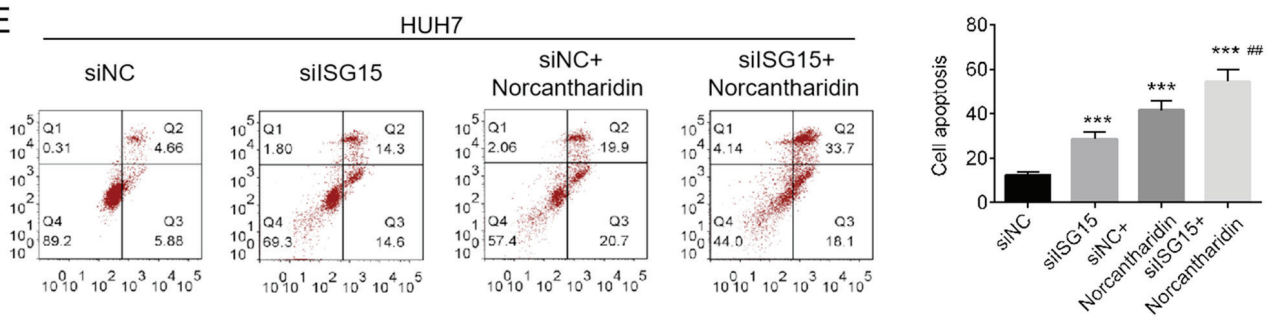

Figure 2. Downregulation of ISG15 increases the sensitivity of hepatocellular carcinoma cells to norcantharidin. Huh7 cells were transfected with siISG15 or siNC, and then treated with norcantharidin. (A) Reverse transcription-quantitative polymerase chain reaction and (B) western blot analysis of ISG15 expression in Huh7 cells, to confirm the transfection efficiency. (C) Proliferation of Huh7 cells was assessed by a Cell Counting Kit-8 assay. (D) Colony formation ability of Huh7 cells was assessed using a colony formation assay. Magnification, x100. (E) Apoptosis of Huh7 cells was examined by Annexin V/PI staining. Data are presented as the mean \pm standard deviation. ${ }^{* * * *} \mathrm{P}<0.001$ vs. siNC group; ${ }^{\# \#} \mathrm{P}<0.01$ vs. siNC + norcantharidin group. ISG15, interferon-stimulated gene 15 ; si, small interfering RNA; NC, negative control; PI, propidium iodide.
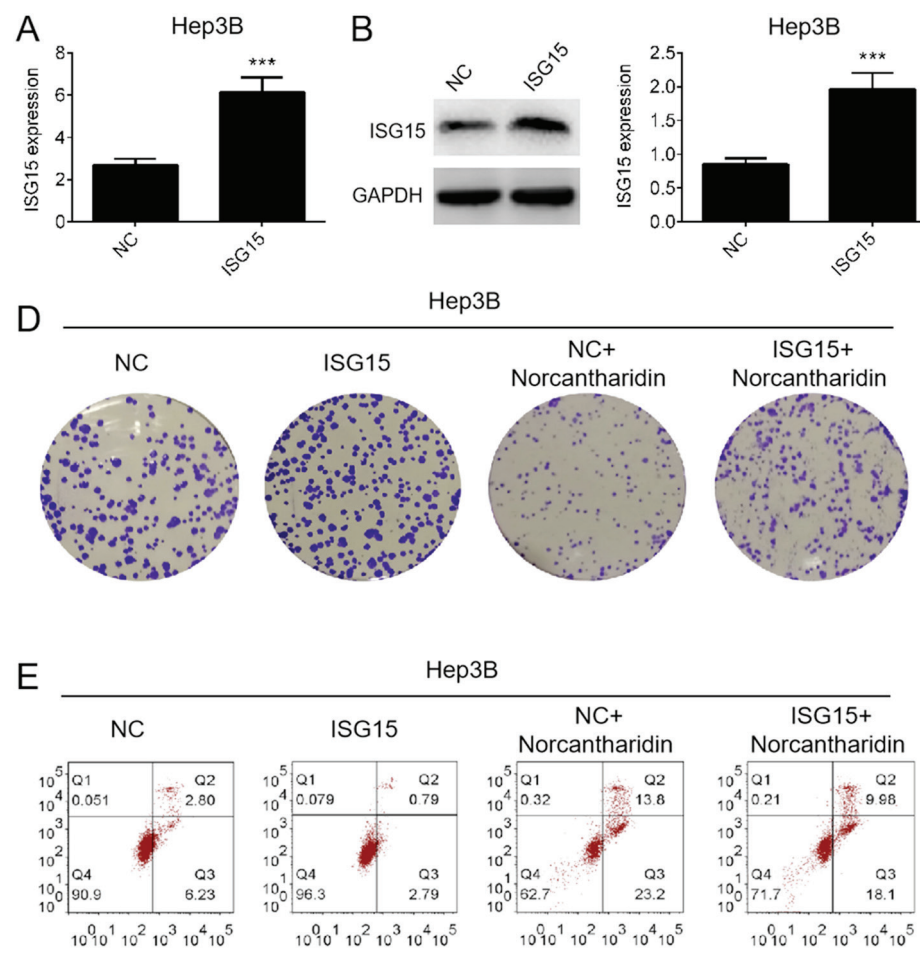

Нер3B

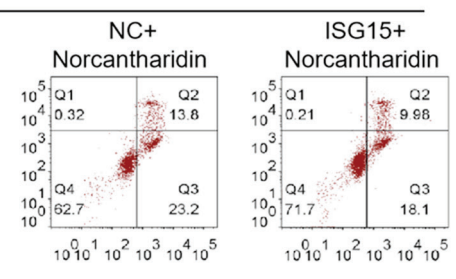

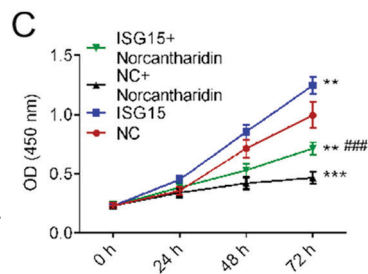
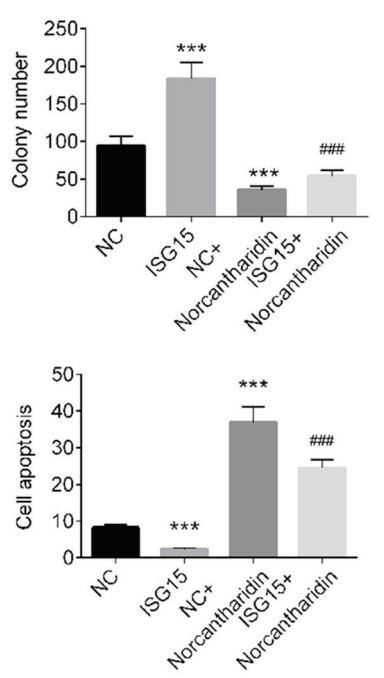

Figure 3. Norcantharidin reverses the tumor-promoting activities of ISG15 on hepatocellular carcinoma cells. Hep3B cells were transfected with ISG15 overexpression or NC plasmid, and then treated with norcantharidin. (A) Reverse transcription-quantitative polymerase chain reaction and (B) western blot analysis of ISG15 expression in Hep3B cells, to confirm the transfection efficiency. (C) Proliferation of Hep3B cells was assessed by a Cell Counting Kit-8 assay. (D) Colony formation ability of Hep3B cells was determined by a colony formation assay. Magnification, x100. (E) Apoptosis of Hep3B cells was

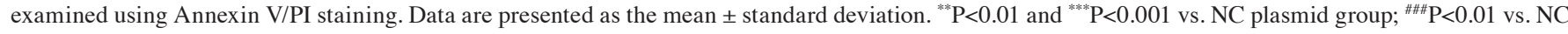
plasmid + norcantharidin group. ISG15, interferon-stimulated gene 15; NC, negative control; PI, propidium iodide. 

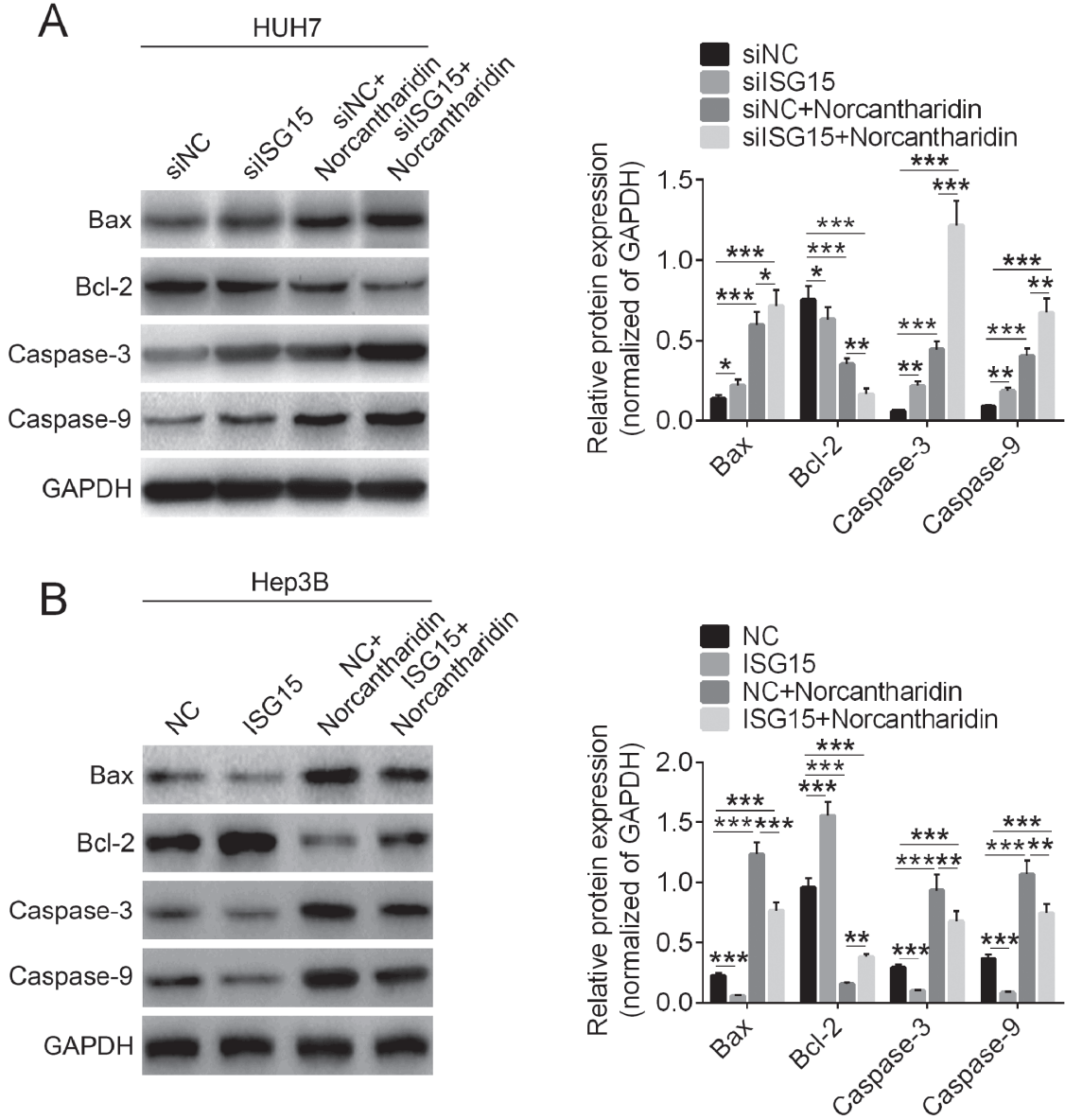

Figure 4. Apoptosis-associated proteins are regulated by ISG15 and norcantharidin. (A) Huh7 and (B) Hep3B cells were treated with ISG15 overexpressing plasmid and norcantharidin, and then Bax, Bcl-2, caspase-3 and caspase- 9 expression levels were determined by western blot analysis. Data are presented as the mean \pm standard deviation. ${ }^{*} \mathrm{P}<0.05,{ }^{* *} \mathrm{P}<0.01$ and ${ }^{* * *} \mathrm{P}<0.001$. ISG15, interferon-stimulated gene $15 ; \mathrm{Bcl}-2$, B-cell lymphoma 2; Bax, Bcl-2-associated X protein.

The results suggest that ISG15 overexpression can reverse the inhibitory effects of norcantharidin on HCC cells.

Apoptosis-associated proteins are regulated by ISG15 and norcantharidin. The expression levels of apoptosis-associated proteins in HCC cells were detected by western blot analysis. As presented in Fig. 4A, treatment with norcantharidin alone significantly increased the protein expression levels of Bax, caspase-3 and caspase-9, and decreased Bcl-2 expression $(\mathrm{P}<0.01)$. Downregulation of ISG15 markedly aggravated the effects of norcantharidin on expression of apoptosis-associated proteins in HCC cells. Furthermore, overexpression of ISG15 was observed to significantly inhibit the expression levels of Bax, caspase-3 and caspase-9, and increase Bcl-2 expression, as compared with the control group $(\mathrm{P}<0.05$; Fig. 4B). Upon combined treatment with the plasmid and norcantharidin, ISG15 overexpression was found to markedly reverse the effect of norcantharidin on the protein expression. These findings suggest that proteins associated with apoptosis were regulated by ISG15 and norcantharidin treatment.

\section{Discussion}

Various studies have reported that regulating prognostic markers increases the sensitivity of malignant tumor cells to chemotherapy drugs, which may result in an important benefit in the treatment of malignant tumors $(26,27)$. The study by Fang et al (28) reported that downregulation of UBC9 increased the sensitivity of HCC cells to doxorubicin. In addition, Meng et al (29) indicated that MEK inhibitors enhance the sensitivity of multidrug resistant HCC cells to chemotherapeutic drugs. Previous studies further reported that norcantharidin suppresses proliferation, inhibits invasion and metastasis, and induces apoptosis in HCC cells, which is a potential treatment of $\mathrm{HCC}(30,31)$.

ISG15 expression has been reported to be increased in breast (14), lung (15) and bladder cancer (16), as well as in HCC (17). Knockdown of ISG15 using siRNAs inhibited xenograft tumor growth and prolonged the survival of tumor-bearing mice with HCC (18). ISG15 is further considered as a potential tumor biomarker for drug sensitivity $(19,20)$. In the present study, the mRNA and protein expression levels of ISG15 in HCC tissues were found to be significantly increased in HCC tissues. Furthermore, according to the previous studies (20-22), the clinicopathological significance of ISG15 levels in patients with HCC was further investigated in the current study. It was observed that ISG15 levels in HCC tissues were significantly correlated with tumor size, TNM stage and differentiation. These results indicated that ISG15 may function as an oncogene in HCC.

Although norcantharidin has evident curative effects, it also exhibits organ toxicity, which limits its clinical 
application to a certain extent (10). Therefore, it is important to increase the sensitivity of HCC cells to norcantharidin. Zhang et al (30) reported that FAM46C serves a critical role in the antiproliferative and proapoptotic effects of norcantharidin in HCC cells. Xiong et al (32) demonstrated that Atg5 siRNA treatment affected cell autophagy and enhanced the anticancer action of norcantharidin through reactive oxygen species generation and the mitochondrial apoptosis pathway. The current study examined how ISG15 downregulation in Huh7 cells and ISG15 upregulation in Hep3B cells affected the proliferation, colony formation ability and apoptosis following norcantharidin treatment. The results indicated that ISG15 knockdown significantly accentuated the antiproliferation and apoptosis-promoting effects of norcantharidin on HCC cells. By contrast, overexpression of ISG15 increased the proliferation and decreased the apoptosis of HCC cells, while norcantharidin notably reversed the effects of ISG15.

The expression levels of apoptosis-associated proteins Bax, Bcl-2, caspase-3 and caspase- 9 were also investigated in the current study. Bcl-2 is an oncogenic protein that inhibits apoptosis, while overexpression of Bax antagonizes the protective effects of Bcl-2 and causes cell death (33). Caspase- 9 is considered to be a caspase initiator, the activation of which subsequently activates caspase-3 to induce apoptosis (34). In the present study, the expression levels of Bax, Bcl-2, caspase-3 and caspase-9 were found to be regulated by siISG15 and/or norcantharidin treatment. The results revealed that ISG15 knockdown sensitized HCC cells to norcantharidin-induced apoptosis by regulating apoptosis-associated proteins.

In conclusion, the present study reported that ISG15 expression was increased in HCC tissues, suggesting that it may serve as a potential prognostic marker in HCC. Furthermore, downregulation of ISG15 effectively sensitized HCC cells to norcantharidin-induced apoptosis; however, the underlying mechanism involved needs to be further studied. The current study may provide novel insights for the clinical application of norcantharidin and molecular targeted therapy for HCC.

\section{Acknowledgements}

Not applicable.

\section{Funding}

No funding was received.

\section{Availability of data and materials}

The datasets used and/or analyzed during the current study are available from the corresponding author on reasonable request.

\section{Authors' contributions}

BXC and CFN collected the clinical samples and performed most of the experiments. SQJ and YSL collected and analyzed data from public datasets, and performed the RT-qPCR assay and statistical analysis. BB and ZL conceived and designed the study, and assisted in the drafting of the manuscript. All authors have read and approved the final manuscript.

\section{Ethics approval and consent to participate}

The Ethics Committee of The First Affiliated Hospital of Soochow University reviewed and approved the study, and written informed consent was obtained from each participant at the examination phase. The study complied with the principles of the Helsinki Declaration.

\section{Patient consent for publication}

Not applicable.

\section{Competing interests}

The authors declare that they have no competing interests.

\section{References}

1. Zakharia K, Luther CA, Alsabbak H and Roberts LR: Hepatocellular carcinoma: Epidemiology, pathogenesis and surveillance-implications for sub-Saharan Africa. S Afr Med J 108: 35-40, 2018

2. Kim WJ, Kim KH, Kim SH, Kang WH and Lee SG: Laparoscopic versus open liver resection for centrally located hepatocellular carcinoma in patients with cirrhosis: A propensity score-matching analysis. Surg Laparosc Endosc Percutan Tech 28: 394-400, 2018.

3. Xie W, Qiao X, Shang L, Dou J, Yang X, Qiao S and Wu Y: Knockdown of ZNF233 suppresses hepatocellular carcinoma cell proliferation and tumorigenesis. Gene 679: 179-185, 2018.

4. Abdel-Rahman $\mathrm{O}$ and Cheung WY: The expanding role of systemic therapy in the management of hepatocellular carcinoma. Can J Gastroenterol Hepatol 2018: 4763832, 2018.

5. Tanaka H, Hijioka S, Iwaya H, Mizuno N, Kuwahara T, Okuno N, Ito A, Kuraoka N, Matsumoto S, Obata M, et al: Fibrolamellar hepatocellular carcinoma with multiple lung metastases treated with multidisciplinary therapy. Intern Med 57: 3537-3543, 2018.

6. Yoon SM, Ryoo BY, Lee SJ, Kim JH, Shin JH, An JH, Lee HC and Lim YS: Efficacy and safety of transarterial chemoembolization plus external beam radiotherapy vs. sorafenib in hepatocellular carcinoma with macroscopic vascular invasion: A randomized clinical trial. JAMA Oncol 4: 661-669, 2018.

7. Choi SH and Seong J: Strategic application of radiotherapy for hepatocellular carcinoma. Clin Mol Hepatol 24: 114-134, 2018.

8. Yang PY, Chen MF, Tsai CH, Hu DN, Chang FR and Wu YC: Involvement of caspase and MAPK activities in norcantharidin-induced colorectal cancer cell apoptosis. Toxicol In Vitro 24: 766-775, 2010.

9. Peng C, Liu X, Liu E, Xu K, Niu W, Chen R, Wang J, Zhang Z, Lin P, Wang J, et al: Norcantharidin induces HT-29 colon cancer cell apoptosis through the alphavbeta6-extracellular signal-related kinase signaling pathway. Cancer Sci 100: 2302-2308, 2009.

10. Yeh CB, Hsieh MJ, Hsieh YH, Chien MH, Chiou HL and Yang SF: Antimetastatic effects of norcantharidin on hepatocellular carcinoma by transcriptional inhibition of MMP-9 through modulation of NF-kB activity. PLoS One 7: e31055, 2012.

11. Sun CY, Zhu Y, Li XF, Tang LP, Su ZQ, Wang XQ, Li CY, Yang HM, Zheng GJ and Feng B: Norcantharidin alone or in combination with crizotinib induces autophagic cell death in hepatocellular carcinoma by repressing c-Met-mTOR signaling. Oncotarget 8: 114945-114955, 2017.

12. Gao Y, Li W, Liu R, Guo Q, Li J, Bao Y, Zheng H, Jiang S and Hua B: Norcantharidin inhibits IL-6-induced epithelialmesenchymal transition via the JAK2/STAT3/TWIST signaling pathway in hepatocellular carcinoma cells. Oncol Rep 38: 1224-1232, 2017.

13. Bektas N, Noetzel E, Veeck J, Press MF, Kristiansen G, Naami A, Hartmann A, Dimmler A, Beckmann MW, Knüchel R, et al: The ubiquitin-like molecule interferon-stimulated gene 15 (ISG15) is a potential prognostic marker in human breast cancer. Breast Cancer Res 10: R58, 2008.

14. Hadjivasiliou A: ISG15 implicated in cytoskeleton disruption and promotion of breast cancer. Expert Rev Proteomics 9: 7, 2012. 
15. Tessema M,Yingling CM, Thomas CL,Klinge DM,Bernauer AM Liu Y, Dacic S, Siegfried JM, Dahlberg SE, Schiller JH and Belinsky SA: SULF2 methylation is prognostic for lung cancer survival and increases sensitivity to topoisomerase-I inhibitors via induction of ISG15. Oncogene 31: 4107-4116, 2012.

16. Andersen JB, Aaboe M, Borden EC, Goloubeva OG, Hassel BA and Orntoft TF: Stage-associated overexpression of the ubiquitin-like protein, ISG15, in bladder cancer. Br J Cancer 94 $1465-1471,2006$

17. Qiu X, Hong Y, Yang D, Xia M, Zhu H, Li Q, Xie H, Wu Q, Liu C and Zuo C: ISG15 as a novel prognostic biomarker for hepatitis B virus-related hepatocellular carcinoma. Int J Clin Exp Med 8: 17140-17150, 2015.

18. Li C, Wang J, Zhang H, Zhu M, Chen F, Hu Y, Liu H and Zhu H: Interferon-stimulated gene 15 (ISG15) is a trigger for tumorigenesis and metastasis of hepatocellular carcinoma. Oncotarget 5: 8429-8441, 2014.

19. Desai SD, Wood LM, Tsai YC, Hsieh TS, Marks JR, Scott GL, Giovanella BC and Liu LF: ISG15 as a novel tumor biomarker for drug sensitivity. Mol Cancer Ther 7: 1430-1439, 2008.

20. Li X, Zhao Q, Qi J, Wang W, Zhang D, Li Z and Qin C: lncRNA Ftx promotes aerobic glycolysis and tumor progression through the PPAR $\gamma$ pathway in hepatocellular carcinoma. Int J Oncol 53 : 551-566, 2018

21. Lin Q, Peng S and Yang Y: Inhibition of CD9 expression reduces the metastatic capacity of human hepatocellular carcinoma cell line MHCC97-H. Int J Oncol 53: 266-274, 2018.

22. Kondo R, Ishino K, Wada R, Takata H, Peng WX, Kudo M, Kure S, Kaneya Y, Taniai N, Yoshida H and Naito Z: Downregulation of protein disulfideisomerase A3 expression inhibits cell proliferation and induces apoptosis through STAT3 signaling in hepatocellular carcinoma. INT J Oncol 54: 1409-1421, 2019.

23. Tan X, Liao Z, Liang H, Chen X, Zhang B and Chu L: Upregulation of liver kinase B1 predicts poor prognosis in hepatocellular carcinoma. Int J Oncol 53: 1913-1926, 2018

24. Iwai N, Yasui K, Tomie A, Gen Y, Terasaki K, Kitaichi T, Soda T, Yamada N, Dohi O, Seko Y, et al: Oncogenic miR-96-5p inhibits apoptosis by targeting the caspase- 9 gene in hepatocellular carcinoma. Int J Oncol 53: 237-245, 2018

25. Livak KJ and Schmittgen TD: Analysis of relative gene expression data using real-time quantitative PCR and the 2(-Delta Delta C(T)) method. Methods 25: 402-408, 2001.
26. Gu WJ and Liu HL: Induction of pancreatic cancer cell apoptosis, invasion, migration, and enhancement of chemotherapy sensitivity of gemcitabine, 5-FU, and oxaliplatin by hnRNP A2/B1 siRNA. Anticancer Drugs 24: 566-576, 2013.

27. Liu T, Xiong J, Yi S, Zhang H, Zhou S, Gu L and Zhou M: FKBP12 enhances sensitivity to chemotherapy-induced cancer cell apoptosis by inhibiting MDM2. Oncogene 36: 1678-1686, 2017.

28. Fang S, Qiu J, Wu Z, Bai T and Guo W: Down-regulation of UBC9 increases the sensitivity of hepatocellular carcinoma to doxorubicin. Oncotarget 8: 49783-49795, 2017.

29. Meng Q, He X, Xie G, Tian Q, Shu X, Li J and Xiao Y: MEK inhibitor enhances sensitivity to chemotherapeutic drugs in multidrug resistant hepatocellular carcinoma cells. Oncol Lett 14: 3089-3095, 2017.

30. Zhang QY, Yue XQ, Jiang YP, Han T and Xin HL: FAM46C is critical for the anti-proliferation and pro-apoptotic effects of norcantharidin in hepatocellular carcinoma cells. Sci Rep 7: 396, 2017.

31. Zhang S, Li G, Ma X, Wang Y, Liu G, Feng L, Zhao Y, Zhang G, Wu Y, Ye X, et al: Norcantharidin enhances ABT-737-induced apoptosis in hepatocellular carcinoma cells by transcriptional repression of Mcl-1. Cell Signal 24: 1803-1809, 2012.

32. Xiong X, Wu M, Zhang H, Li J, Lu B, Guo Y, Zhou T, Guo H, Peng R, Li X, et al: Atg5 siRNA inhibits autophagy and enhances norcantharidin-induced apoptosis in hepatocellular carcinoma. Int J Oncol 47: 1321-1328, 2015.

33. Zhao G, Zhu Y, Eno CO, Liu Y, Deleeuw L, Burlison JA, Chaires JB, Trent JO and Li C: Activation of the proapoptotic Bcl-2 protein Bax by a small molecule induces tumor cell apoptosis. Mol Cell Biol 34: 1198-1207, 2014.

34. Kim B, Srivastava SK and Kim SH: Caspase-9 as a therapeutic target for treating cancer. Expert Opin Ther Targets 19: 113-127, 2015.

(c) (i) () $($ This work is licensed under a Creative Common Attribution-NonCommercial-NoDerivatives 4.0 International (CC BY-NC-ND 4.0) License. 